\title{
Conservative decisions guided by the anterior cingulate cortex
}

\author{
Nicholas Myers* and Valentin Wyart \\ Department of Experimental Psychology, University of Oxford, Oxford, UK \\ *Correspondence: nicholas.myers@psy.ox.ac.uk
}

\section{A commentary on}

Decision threshold modulation in the human brain

by Domenech, P., and Dreher, J. C. (2010). J. Neurosci. 30, 14305-14317.

When faced with a visual stimulus, how does our brain process sensory information to decide which action to take? Only in the last 10 years have researchers begun to unravel the neural bases of such perceptual decisions (Heekeren et al., 2004; Gold and Shadlen, 2007). A popular theory holds that dedicated neural circuits achieve this by accumulating evidence from the environment until they reach a threshold and commit to a decision (such as whether a briefly presented shape is a triangle or a circle). Tasks have classically been designed such that each decision is independent of past decisions, and so the accumulation of information starts anew at the beginning of each trial. However, in ordinary situations, we use prior experience to help us decide. Expecting an upcoming stimulus to have a particular shape should therefore make the next decision easier and faster. While the representation of expectations in the brain has been related to faster reaction times (Summerfield and Koechlin, 2008), the neural mechanisms underlying this behavioral advantage remain unclear. Theoretical decision-making models have proposed two ways to produce this effect, either (1) by lowering the decision threshold (Carpenter and Williams, 1995), or (2) by accelerating the accumulation rate of sensory evidence. Recent behavioral evidence suggests that both may occur in parallel (Liston and Stone, 2008).

Recently, Domenech and Dreher (2010) addressed this issue by testing how varying amounts of predictive information influence perceptual decisions, and used functional magnetic resonance imaging (fMRI) to test where in the brain this influence may occur. In their task, subjects responded to target shapes (e.g., triangles) appearing in a stream of distractor shapes. The authors varied the likelihood with which any particular stimulus (or sequence of two stimuli) would be followed by a target. Since participants were not told about these regularities (and indeed failed to notice them during the experiment), predictive information was learned implicitly during each block.

In an elegant analysis of reaction time distributions (Carpenter and Williams, 1995), the authors were able to test whether predictive information influenced the decision threshold, the accumulation rate of sensory evidence, or both. They found that more predictive information lowered the decision threshold but did not accelerate the rate of evidence accumulation, biasing participants to make faster responses when they were expecting a target shape on the basis of previous distractors. However, since all stimuli were easily discriminable, perceptual processes had likely reached asymptotic performance and could not be further improved by gain control mechanisms. When stimuli are harder to discriminate, predictive information may influence perceptual processing as well (Liston and Stone, 2008; Melloni et al., 2011).

If predictive information lowers the decision threshold, any evidence-accumulation brain region should respond less to targets when predictive information is high, but should respond more when the accumulation rate is fast. Only the right dorsolateral prefrontal cortex (dlPFC) had both response properties (their Figure 7), partially confirming previous findings in humans and monkeys that the dlPFC represents the amount of accumulated sensory evidence (Heekeren et al., 2004; Gold and Shadlen, 2007). Predictive information itself was negatively correlated with a large, distributed network. It consisted of the inferior frontal gyri, the right intra-parietal sulcus, the left and right dlPFC, and, most importantly, the anterior cingulate cortex (ACC, their Figure 5). In this network, only the ACC responsiveness to predictive information was related to how much participants used that information to make faster decisions. In other words, while a large fronto-parietal network was involved in tracking predictive information, the ACC seemed to have a special role in using this information to set the decision threshold. Furthermore, when predictive information needed to be tracked further back into the past to be useful, the coupling between the ACC and parts of the right dlPFC increased (their Figure 8). The authors ensured that predictive information was not correlated with other measures that could have influenced ACC activity, such as error likelihood, prediction-error magnitude, entropy, or overall target probability (their Figure 6).

The authors claim that the ACC is involved in the "contextual guidance of the decision process" by changing the decision threshold in response to the amount of predictive information. Although their results support a model in which ACC reacts to recent stimulus history, it is unclear how the ACC actually implements this contextual guidance, or whether it is truly involved in changing the decision threshold. Their main finding indicates that the ACC tends to "turn off" when we are able to rely on predictive information, whereas its activity increases when we have little predictive information and therefore need to rely more on the current sensory input to make an accurate decision. This result mirrors a recent finding in a study on reward-guided decision-making by Behrens et al. (2007), in which they showed that increased volatility (i.e., a decrease in the stability of contextual contingencies) correlates with ACC activity at long time scales. When volatility is high, Behrens et al. have shown that human subjects give more weight to the immediate reward history by increasing their learning rate, and that this increase in learning rate 
correlates with increased ACC activity. The results obtained by Domenech and Dreher (2010) show that the ACC can also track changes in predictive information at much faster time scales.

Together, the results indicate that the ACC is recruited for maintaining a conservative decision threshold whenever the past provides little predictive information. It may have been interesting to see whether the ACC also shows an increased response when predictive information is high, but no target appears. Indeed, by showing that ACC activity correlates with increases in decision threshold following disconfirmations of predictive information, Domenech and Dreher (2010) could have made an even stronger case for the hypothesis that the ACC is involved in threshold regulation. Furthermore, different brain regions may represent predictive information over different time scales. The dlPFC may react on the shortest time scale by integrating sensory evidence of the current stimulus set. The ACC may respond to higher-order predictive information over slightly longer intervals. Contextual information may be held over even longer time scales in ventromedial prefrontal cortex (Koechlin and Summerfield, 2007). By integrating the influence of expectation into a computational model and by testing its neural bases, Domenech and Dreher (2010) have helped to formalize the influence of memory on perceptual decision-making.

\section{ACKNOWLEDGMENTS}

We would like to thank Christopher Summerfield and Anna C. Nobre for helpful comments.

\section{REFERENCES}

Behrens, T. E., Woolrich, M. W., Walton, M. E., and Rushworth, M. F. (2007). Learning the value of information in an uncertain world. Nat. Neurosci. $10,1214-1221$.

Carpenter, R., and Williams, M. (1995). Neural computation of log likelihood in control of saccadic eye movements. Nature 377, 59-62.

Domenech, P., and Dreher, J. C. (2010). Decision threshold modulation in the human brain. J. Neurosci. 30 14305-14317.
Gold, J. I., and Shadlen, M. N. (2007). The neural basis of decision making. Annu. Rev. Neurosci. 30, 535-574.

Heekeren, H. R., Marrett, S., Bandettini, P. A., and Ungerleider, L. G. (2004). A general mechanism for perceptual decision-making in the human brain. Nature 431, 859-862.

Koechlin, E., and Summerfield, C. (2007). An information theoretical approach to prefrontal executive function. Trends Cogn. Sci. (Regul. Ed.) 11, 229-235.

Liston, D. B., and Stone, L. S. (2008). Effects of prior information and reward on oculomotor and perceptual choices. J. Neurosci. 28, 13866-13875.

Melloni, L., Schwiedrzik, C. M., Müller, N., Rodriguez, E. and Singer, W. (2011). Expectations change the signatures and timing of electrophysiological correlates of perceptual awareness. J. Neurosci. 31, 1386-1396.

Summerfield, C., and Koechlin, E. (2008). A neural representation of prior information during perceptual inference. Neuron 59, 336-347.

Received: 28 February 2011; accepted: 14 April 2011; published online: 27 April 2011.

Citation: Myers N and Wyart V (2011) Conservative decisions guided by the anterior cingulate cortex. Front. Hum. Neurosci. 5:44. doi: 10.3389/fnhum.2011.00044

Copyright (c) 2011 Myers and Wyart. This is an openaccess article subject to a non-exclusive license between the authors and Frontiers Media SA, which permits use, distribution and reproduction in other forums, provided the original authors and source are credited and other Frontiers conditions are complied with. 